Vargas N. y cois.

Rev. Chll. Pediatr. 65 (5); 264-267, 1994

\title{
Enfermedades crónicas en pacientes pediátricos hospitalizados: frecuencia y tipo de enfermedad
}

\author{
Nelson A. Vargas C.'; Orquídea Arredondo B.'; Gianinna Ilabaca P.'; \\ Andrés Manurana P.' ; Gustavo Ortuvia C. ${ }^{1}$
}

\section{Chronic diseases among hospitalized children: frequency and type of illness}

\begin{abstract}
Frequency and rype of illness were studied in patients under titteen years of age, dischorged from the pedialric wards of o university associoled public hospilal al melropotilan Santiago, Chile. From april through sepiember 1992, patients discharged from hospital sections for infants, infectious diseases, child surgery, critical care and medicine were classified as acule, confirmed chronic or unconfirmed chraric disease. Oncohematologic, multiple trouma and emergency cases were excluded. Diagnosis given by treaking physician were recorded and any illness lasting over six months was defined as a chronic disease. Chronicity reached $35.2 \%$ among whole hospital discharges and $32.9 \%$ among children discharges, increasing gradually according to age to reach $47.1 \%$ in children aged 10 years or more. Unconfirmed diagnasis among 137 chronic children reacred $13.1 \%(\mathrm{n}=18)$. Higher uncertainty in diagnosis was recorded under six months of age $(25 \%)$. Median age at hospital cischarge was 18 months in children with chronic diseases and only 10 months in acule coses. Most common groups of diagnosis were respiratory infections, congenilal abnormalities and genitourinary tract diseases.
\end{abstract}

(Koy words: chronic diseoses, children morbidity. health care.)

El descenso de la mortalidad infantil en Chile ha aumentado la esperanza de vida de niños que antes tenían escasas posibilidades de sobrevivir. Muchos de ellos, a pesar de hacerlo, no curan completamente o quedan con secuelas, originadas ya sea por la condición que los puso en riesgo o por los procedimientos médicos a que fueron sometidos para salvarles. Estas razones han originado un aumento, mal cuantificado e insuficientemente estudiado, de enfermedades crónicas en los niños, cuyos casos sobrepasarían los 500 000, según un informe hecho en 1991 por el Connté de Enfermedades Crónicas de la Sociedad Chilena de Pediatría ${ }^{1}$. Las estadísticas acerca de estas afecciones -que suman, a su impacto médico, elevados costos económicos y sociales- son escasas. La importancia de conocer el problema radica en la necesidad de adecuar la atención de salud y la educación médica a esta nueva realidad.

1. Departamento de Pediatría y Cirngía Infantil Occidente. Departamento de Salud Pública Occidente. Facultad de Medicina. Universidad de Chile.
El propósito de este estudio fue medir la frecuencia y el tipo de enfermedades crónicas en menores de 15 años, admitidos en un hospital docente de la región metropolitana. Los objetivos fueron precisar la proporción de egresados con diagnósticos crónicos (de certeza y de sospecha); así como la proporción con algunos diagnósticos seleccionados.

\section{Material y Método}

Se consignaron los egresos de los sectores de lactantes, infecciosos, cirugía infantil, cuidado intensivo y niños mayores, entre el 1 de abril y el 30 de setiembre de 1992, en el Servicio de Pediatría del Hospital San Juan de Dios, de Santiago. Este es el hospital base del Servicio de Salud Metropolitano Occidente. En el servicio pediálrico no exisIe una unidad de tratamiento intensivo completa, lo que debe ser considerado al analizar los resultados. No se incluyeron los casos oncohematológicos (278 egresos) porque, en opinión de algunos oncólogos pediátricos, no son crónicos propiamente tales. El estudio excluyó a politraumatizados y enfermedades de urgencia, pues el servicio que los atiende en el área está en otro hospital.

Para cada menor de 15 a jos se consignaron los diagnósticos de la epicrisis (asignados según criterio del médico tratante), separando los casos crónicos para un análisis 
más datallado. Se confeccionó una lista de afecciones crónicas, usando la Clasiticación Internacional de Enfermedades de la OMS. IX revisión ${ }^{2}$, para agrupar los casos seleccionados. Los casos dudosos se determinaron en reunjón del grupo investigador, asesorado por especialistas y lextos. Según la scguridad del diagnóstico. los casos se clasificaron en: de ceneza (clinica y laboratorio compatibles con el diagnostico) y de sospecha (clínica sugerente, sin conírmación de laboratorio). Se consideró enfermedad crónica cualquiera que durase más de seis meses, sin ser sinónima de incurable.

\section{Resultados}

En los seis meses del estudio hubo 469 egresos de 416 niños. De estos últimos, 137 pacientes fueron catalogados como crónicos y ellos originaron 165 egresos (1,2 egresos por niño). Fueron clasificados como agudos $279 \mathrm{ni}$ ños, que originaron 304 egresos ( 1,09 por niño). La proporción de crónicos fue $35,2 \%$ entre los egresos y 32,9\% entre los niños (tabla 1). La mediana de la edad en los casos crónicos fue 19 meses y en los agudos 10 meses. La proporción de egresos con enfermedades crónicas aumentó gradualmente con la edad de los niños, hasta acercarse a $50 \%$, en los mayores de 10 años (tabla 2). Las mujeres representaban $42,3 \%(\mathrm{n}=118)$ de los pacientes con problemas agudos y $48,9 \%(n=67)$ de los crónicos.

Entre los 137 niños con enfermedades crónicas. $13.1 \%(\mathrm{n}=18)$ tenían sólo diagnóstico de sospecha, destacando la mayor incertidumbre en el diagnóstico bajo los scis meses (tabla 3). Las afecciones respiratorias conformaron el grupo más frecuente de diagnóstico entre las en. fermedades crónicas de esta serie, seguidas de Las anomalías congénitas y las afecciones génitourinarias (tabla 4).

\section{Comentario}

El proceso de catalogar a un niño como portador de una enfermedad crónica no es siempre fácil y se afecta con las capacidades resolutivas y de diagnóstico de los servicios de salud que. de esta manera, condicionan sus resultados. Por lo tanto, configurar un panorama nacional del problema exige estudios en otros ámbitos. Para comprender los hallazgos de este trabajo hay que tener presente las exclusiones que se han detallado y la condición de centro de referencia
Tabla 1

Proporción de crónicos entre egresos y pacientes

\begin{tabular}{lrrr} 
& \multicolumn{1}{c}{ n } & \multicolumn{1}{c}{$\%$} \\
\hline Egresos & 469 & 100,0 & \\
$\quad$ Crónicos & 165 & 35,2 & \\
Agudos & 304 & 64,8 & \\
& & & \\
Pacientes & 416 & 100,0 & 100,0 \\
$\quad$ Crónicos tolales & 137 & 32,9 & 86,9 \\
Crónico de certeza & 119 & & 13,1 \\
Sospecha cronicidad & 18 & & \\
Agudos & 279 & 67,1 & \\
\hline
\end{tabular}

Tabla 2

Proporción de pacientes crónicos en las distintas edades

\begin{tabular}{|c|c|c|c|}
\hline \multirow[t]{2}{*}{ Edad } & \multirow{2}{*}{$\begin{array}{c}\text { Total } \\
\mathbf{n}\end{array}$} & \multicolumn{2}{|c|}{ Crónicos } \\
\hline & & n & $\%$ \\
\hline \multicolumn{4}{|c|}{ Menores de 1 año } \\
\hline $\begin{array}{l}<3 \mathrm{~m} \\
3 \text { a } 5 \mathrm{~m} \\
6 \text { a } 11 \mathrm{~m} \\
\text { Subtotal }\end{array}$ & $\begin{array}{r}72 \\
45 \\
79 \\
196\end{array}$ & $\begin{array}{r}12 \\
9 \\
28 \\
49\end{array}$ & $\begin{array}{l}16,7 \\
19.1 \\
35,4 \\
21,9\end{array}$ \\
\hline \multicolumn{4}{|c|}{ Mayores de 1 año } \\
\hline $\begin{array}{l}1 \text { ano } \\
2 \text { a } 5 \\
5 \text { a y } \\
10 \text { a } 14 \\
15 \text { y más }\end{array}$ & $\begin{array}{r}79 \\
48 \\
40 \\
51 \\
2\end{array}$ & $\begin{array}{r}30 \\
18 \\
16 \\
24 \\
0\end{array}$ & $\begin{array}{r}38,0 \\
37,5 \\
40,0 \\
47,1 \\
-\end{array}$ \\
\hline Subtotal & 220 & 88 & 40,0 \\
\hline Total & 416 & 137 & 32,9 \\
\hline
\end{tabular}

nefrologica del servicio en que se hizo el análisis.

La proporción de enfermos crónicos encontrada confirma resultados previos ${ }^{3}$ y no parece modificable en el futuro cercano. Las razones de esta alta frecuencia son, posiblemente, la eficacia de los programas destinados a reducir las hospitalizaciones por enfertnedades agudas y el probable aumento de las enfermedades crónicas, por supervivencia de niños que antes fallecían. Como es natural, se observo aumento de la proporción de afecciones crónicas con el de la edad, pues ésta permite mayor expresión de la enfermedad y, por lo tanto, más precisión en el diagnóstico. 
Tabla 3

Diagnósticos de sospecha y certeza por edad de los pacientes crónicos

\begin{tabular}{|c|c|c|c|c|c|}
\hline \multirow[t]{2}{*}{ Edad } & \multirow{2}{*}{$\begin{array}{l}\text { Crónica total } \\
\text { n }\end{array}$} & \multicolumn{2}{|c|}{ Certezn } & \multicolumn{2}{|c|}{ Sospecha } \\
\hline & & $\mathbf{n}$ & $\%$ & $\mathbf{n}$ & $\%$ \\
\hline$<3 \mathrm{~m}$ & 12 & 9 & 75,0 & 3 & 25,0 \\
\hline 3 a $5 \mathrm{~m}$ & 9 & 6 & 66,6 & 3 & 33,3 \\
\hline 6 a $11 \mathrm{~m}$ & 28 & 27 & 96,4 & 1 & 3,6 \\
\hline 1 aดิ & 30 & 28 & 93,3 & 2 & 6,7 \\
\hline 2 a 5 affos & 18 & 14 & 87,8 & 4 & 22,2 \\
\hline 5 a 9 años & 16 & 13 & 81,2 & 3 & 18,8 \\
\hline 10 a 14 & 24 & 22 & 91,7 & 2 & 8,3 \\
\hline Total & 137 & 119 & 86,9 & 18 & 13,1 \\
\hline
\end{tabular}

Las consecuencias del incremento de enfermedades crónicas en el ambiente intrahospitalario se hacen sentir en nuevas necesidades en al menos cuatro aspectos: apoyo y educación a los padres; generación de normas especiales de manejo intrahospitalario; tecnología adecuada para diagnosticar, tratar y rehabilitar a los afectados, y cambios en los contenidos de los programas de formación de pregrado y posgrado.

La literatura nacional describe pocos esfuerzos de los servicios de salud para apoyar a las familias de los crónicos; las que deben desarrollar estrategias para neutralizar el impacto emocional y económico de la enfermedad. Esta carencia puede atribuirse a la lentitud de los servicios para adaptarse a realidades nuevas y, probablemente, a la falta de recursos.

En lo que respecta al manejo intrahospitalario, conviene subrayar la necesidad de evaluar periódicamente el estado de la nutrición, el desarrollo psicomotor, el cumplimiento de los programas de inmunización y el aporte de nutrientes específicos y estimulación en estos pacientes.

Por otra parte, mientras los hospitales públicos no superen sus problemas de recursos no será posible solucionar las demandas de tecnología necesaria para lograr diagnósticos de mayor precision y, por lo tanto, la proporción de diagnósticos de sospecha se mantendrá alta, en especial si recordamos que el manejo de las enfermedades crónicas obliga a ocupar amplia variedad de métodos, algunos caros, para grupos a veces pequeños de pacientes, lo que le resta prioridad en el momento de asignar recursos.
Es conveniente que la formación pediátrica de pregrado capacite, entre otros aspectos, para el manejo de la patología prevalente en el momento en que se aprende, $y$ en ambientes no especializados, lo que obliga a sacar del hospital una parte importante de las actividades docentes, para desarrollarlas preferentemente en el nivel primario y comunitario de las prestaciones médicas. Entre las dificultades para este cambio se incluyen la necesidad de capacitar al nivel primario asistencial para la docencia y el hecho

\section{Tabla 4}

Grupos de diagnósticos en pacientes crónicos y proporción de certeza según grupo

\begin{tabular}{llll}
\hline Grupo Total & \multicolumn{2}{c}{ Certeza } \\
& n $\%$ & n $\%$
\end{tabular}

Enfermedades intestinales (001-009)

Tuberculosis

(010-018)

Otras infec.y parasit.

(020-139)

Tumores malignos

(140-208)

Tumores benignos y no esp

(210-239)

Eadocrin, nutric, metab, inmunit. (240-279)

Sangre y hematopoyesis

(280-289)

Mentales

(290-319)

S. nervioso y sentidos

(320-389)

Ap. circulatorio

(390-459)

Ap. respiratorio

(460-5]9)

Ap. digestivo

(520-579)

Ap. génitourinario

(580-629)

Piel y tej. celular

(680-709)

Osteorousculares y t. conjuntivo (710-739)

Congénitas

(740-759)

Período perinatal

(760-779)

Mal definidas

(780-799)

Traumatismos, enven. y viol. (800-999)

8

2,25

62,5

$10,3 \quad 100,0$

$\begin{array}{llll}6 & 1,7 & 6 & 100,0\end{array}$

$\begin{array}{llll}2 & 0.6 & 2 & 100,0\end{array}$

$\begin{array}{llll}4 & 1,1 & 4 & 100,0\end{array}$

$29 \quad 8,2 \quad 21 \quad 72,4$

$14 \quad 4,0 \quad 11 \quad 78,6$

$\begin{array}{llll}3 & 0,9 & 3 & 100,0\end{array}$

$\begin{array}{llll}32 & 9,1 & 28 & 87,5\end{array}$

$\begin{array}{llll}13 & 3,7 & 12 & 92,3\end{array}$

$\begin{array}{llll}73 & 20,7 & 65 & 89,0\end{array}$

$\begin{array}{llll}34 & 9,6 & 26 & 52,0\end{array}$

$\begin{array}{llll}50 & 14,2 & 46 & 92,0\end{array}$

$\begin{array}{llll}4 & 1,1 & 3 & 75,0\end{array}$

$\begin{array}{llll}4 & 1,1 & 3 & 75.0\end{array}$

$\begin{array}{llll}55 & 15,6 & 55 & 100,0\end{array}$

$\begin{array}{llll}3 & 0,9 & 2 & 66,6\end{array}$

1 I $3,1 \quad$ II $\quad 100,0$

\begin{tabular}{llll}
\hline Total & 352 & 306 & 86.9
\end{tabular}


que el cuerpo académico - a menudo constituido por especialistas- suele ser renuente a salir del hospital. La especialización pediátrica, a la inversa, debe considerar la frecuencia de las enfermedades crónicas al plantear sus objetivos y actividades, para mantenerse acorde con la evolución de la morbilidad de la población infantil que requiere atención más especializada.

\section{Resumen}

Se estudió la frecuencia y tipo de enfermedades crónicas en menores de 15 años que egresaron de las unidades de lactantes, infecciosos, cirugía infantil, cuidado intensivo y niños mayores de un servicio de pediatría en un hospital docente de Santiago metropolitano, entre el 1 de abril y 30 de setiembre de 1992. No se incluyeron los casos oncobematológicos, de pacientes politraumatizados o de urgencia y se consideró enfermedades crónicas cualquiera que durase más de seis meses. Los casos se clasificaron según la seguridad con que se formuló el diagnóstico, en certeza y sospecha. La proporción de afecciones crónicas fue $35,2 \%$ entre los egresos y $32,9 \%$ entre los niños, aumentando gra- dualmente con la edad, hasta $47,1 \%$ en los mayores de 10 años. Entre los 137 niños con enfermedades crónicas, $13,1 \%(\mathrm{n}=18)$ tenían sólo diagnóstico de sospecha, siendo mayor la incertidumbre en los menores de seis meses $(25 \%)$. La mediana de la edad de los casos crónicos fue 19 meses y solo 10 meses en los agudos. Las afecciones respiratorias conformaron el grupo de diagnósticos más frẹcuente, seguidas de las anomalías congénitas y las afecciones génitourinarias.

(PaJabras clave: enfermedades crónicas, morbilidad infantil, atención de salud.)

\section{Referencias}

1. Anónimo: Enfermedades Crónicas en la Infancia. Chile. 1990. Elernentos para una cuantificación del problema. Sociedad Chilena de Pediatría. Comité de Enfermedades Crónicas. Fondo de las Naciones Unidas para la Infancia. Abril 1991. Santiago.

2. Anonimo: Manual de Clasificación Estadística Internacional de Enfermedades, Traumatismos y Causas de Defunción. Organización Mundial de la Salud. Revisión 1975. Publicación Científica No 353. OPS. Washington. 1978

3. Vargas $N$. Vadell A, Errázuriz $G$ et al.: Enfermedades crónicas en la infancia: aproximación a la medición de su impacto. Rev Chil Pediatr 1990, 61: 337-345 